\title{
Propagative oscillations in co-directional polariton waveguide couplers
}

\author{
J. Beierlein, ${ }^{1, *}$ E. Rozas ${ }^{\dagger},{ }^{2}$ O. A. Egorov,${ }^{3}$ M. Klaas, ${ }^{1}$ A. Yulin, ${ }^{4}$ H. Suchomel,,${ }^{1}$ T.H. Harder,${ }^{1}$ M. Emmerling, ${ }^{1}$ M. \\ D. Martín, ${ }^{2}$ I.A. Shelykh, ${ }^{5,6}$ C. Schneider, ${ }^{1,7}$ U. Peschel, ${ }^{3}$ L. Viña, ${ }^{2,8, \ddagger}$ S. Höfling, ${ }^{1,9}$ and S. Klembt ${ }^{1, \S}$ \\ ${ }^{1}$ Technische Physik, Wilhelm-Conrad-Röntgen-Research Center for Complex Material Systems, \\ and Würzburg-Dresden Cluster of Excellence ct.qmat, \\ Universität Würzburg, Am Hubland, D-97074 Würzburg, Germany \\ ${ }^{2}$ Departamento de Física de Materiales, Instituto Nicolás Cabrera, \\ Universidad Autónoma de Madrid, 28049 Madrid, Spain \\ ${ }^{3}$ Institute of Condensed Matter Theory and Optics Friedrich-Schiller-Universität \\ Jena, Max-Wien-Platz 1, D-07743 Jena, Germany \\ ${ }^{4}$ National Research University of Information Technologies, \\ Mechanics and Optics (ITMO University), Saint-Petersburg 197101, Russia \\ ${ }^{5}$ Faculty of Physics and Engineering, ITMO University, 197101 St. Petersburg, Russia \\ ${ }^{6}$ Science Institute, University of Iceland, IS-107 Reykjavik, Iceland \\ ${ }^{7}$ Institute of Physics, University of Oldenburg, D-26129 Oldenburg, Germany \\ ${ }^{8}$ Instituto de Física de la Materia Condensada, Universidad Autónoma de Madrid, 28049 Madrid, Spain \\ ${ }^{9}$ SUPA, School of Physics and Astronomy, University of St Andrews, St Andrews KY16 9SS, United Kingdom
}

\begin{abstract}
We report on novel exciton-polariton routing devices created to study and purposely guide lightmatter particles in their condensate phase. In a co-directional coupling device, two waveguides are connected by a partially etched section which facilitates tunable coupling of the adjacent channels. This evanescent coupling of the two macroscopic wavefunctions in each waveguide reveals itself in real space oscillations of the condensate. This Josephson-like oscillation has only been observed in coupled polariton traps so far. Here, we report on a similar coupling behavior in a controllable, propagative waveguide-based design. By controlling the gap width, channel length or the propagation energy, the exit port of the polariton flow can be chosen. This co-directional polariton device is a passive and scalable coupler element that can serve in compact, next generation logic architectures.
\end{abstract}

Photonic circuits rely on a variety of fiber-based optical elements for their functionality, which allow easy routing and filtering of the signals; the main drawback of purely photonic schemes for logic operations, however, is a lack of self interaction for very efficient switching ${ }^{1}$ 2 . The remarkable advances in exciton-polariton physics are a result of the progressing control of high-quality microcavities, in which quantum well (QW) excitons and cavity photon modes couple strongly to form new hybrid light-matter eigenstates ${ }^{3}$. Polaritons exhibit a condensate regime at higher densities ${ }^{4}$ with emission properties similar to those of a traditional laser, without having to rely on population inversion ${ }^{5}$. This macroscopic quantum state ${ }^{6,7}$, can propagate over macroscopic distances for high-quality samples ${ }^{8}$. Furthermore, polaritons can be excited, confined and therefore guided in waveguide structures $^{9}$. Propitiously, the excitonic fraction of the polariton condensate is responsible for the observation of strong nonlinear interaction effects ${ }^{10-12}$, the photonic fraction allows for typical photonic benefits like a fast propagation velocity. Due to this interplay, a variety of next generation devices based on polaritons can be envisioned ${ }^{13}$. Especially the possibility to use polaritons

\footnotetext{
$\dagger$ J. Beierlein and E. Rozas contributed to this work equally. * Electronic address: johannes.beierlein@uni-wuerzburg.de $¥$ Electronic address: luis.vina@uam.es

$\S$ Electronic address: sebastian.klembt@physik.uni-wuerzburg. de
}

as information carriers in logic architectures has been addressed theoretically ${ }^{14}$ and experimentally ${ }^{15-22}$. Recently, these ideas have been rekindled by related room temperature experiments using perovskites ${ }^{23}$ and organics materials ${ }^{24}$.

Basic routing effects have been predicted and achieved for polaritons ${ }^{25,26}$, which show some functionality but are mainly based on active optical control. To this end, we demonstrate a new polariton device in a codirectional router, harnessing a Josephson-like oscillation effect in real space, which could feasibly be scaled and does not need active external control. Josephson oscillations ${ }^{27}$ occur when two quantum states are coupled by a transmissive barrier and were first demonstrated in superconductors $^{28}$. Similar effects have been observed in atomic Bose Einstein condensates ${ }^{29-34}$ for which the interaction between the particles is crucial $^{35}$ to observe different interaction dependent regimes of coupling. For polaritons this effect has first been observed in a naturally occurring disorder double potential well ${ }^{36}$ and later in a dimer micropillar arrangement ${ }^{37,38}$.

Our new coupler device consists of extended onedimensional channels which allow observation of the oscillations in the spatial domain, via a specifically tailored fabrication technique.

In this work, dedicated to a proof of concept, we use high-quality GaAs-based microcavities, benefiting from mature fabrication techniques ${ }^{39}$. For the co-directional couplers with waveguide coupling achieved by partially etched mirrors we use molecular beam epitaxially grown 

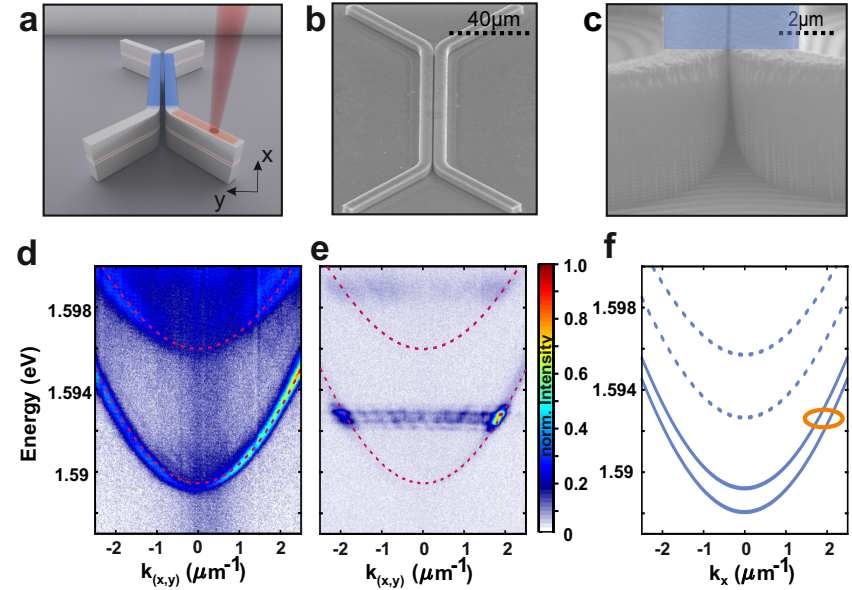

FIG. 1: (a) Device schematic with indicated laser excitation (red), incoupler region (orange) and the coupling region (blue) along the $x$ axis. (b) Top view SEM image of a co-directional polariton coupler. (c) Zoom-in highlighting the coupling region (blue) and the gap between the two waveguides. Here, the cavity and a varying number of mirror pairs are still intact. (d) Measured waveguide dispersion below and (e) above polariton condensation threshold at the input port region (orange) parallel to the waveguide,angled $45^{\circ}$ to the $x$ axis. (f) Calculated dispersion below condensation threshold at the coupling region (blue)

microcavities hosting twelve GaAs quantum wells resulting in a Rabi splitting of $13.9 \mathrm{meV}$ and a quality factor of $Q \approx 5000$ (see Supplemental Material for further details). Sample processing was done via a specially developed reactive ion etching (RIE) process. The first step consists of an electron beam exposure of a polymethyl methacrylate photoresist and subsequent development. Later, a metal layer of calibrated thickness is evaporated on the sampled followed by a lift-off process. After the lift-off process the sample is etched. As a consequence of the protection by the predefined metal layers, the sample is only etched at the exposed positions. Due to the proximity of the structures and the anisotropic etching behaviour of RIE, the etching rate between the waveguides is slower, leaving a certain number of mirror pairs untouched. These left-over mirror pairs between the waveguides facilitate evanescent, photonic coupling. The area around the coupler is nearly etched through the bottom DBR and therefore facilitates strong photonic confinement.

Fig. 1(a) shows a sketch of the intended structure, highlighting an excitation scheme (red), the incoupler region (orange) and the propagation direction $x$ along the coupling area (blue). A scanning electron microscopy image of the full device as well as a zoomed view of the coupling region, stressing the fabrication-induced narrow gap between the structures, are presented in Fig. 1(b) and (c), respectively. The incoupler, which is angled $45^{\circ}$ to the propagation direction $x$ (see Fig. 1(a)), has a width of $2 \mu \mathrm{m}$ and a length of $40 \mu \mathrm{m}$. Figs. 1(d) and (e) depict the energy dispersion of the ground state along the in-
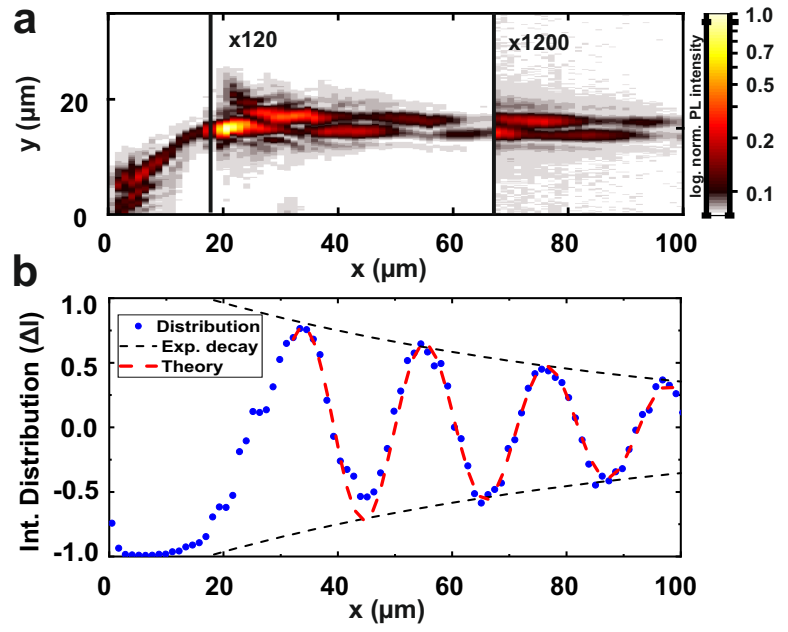

FIG. 2: (a) Energy-resolved real space PL for a coupler device with a gap size of $200 \mathrm{~nm}$ and a coupler region length of $100 \mu \mathrm{m}$ at $E=1.5924-1.5930 \mathrm{eV}$. Polaritons are injected non-resonantly in the lower left incoupler and exhibit a distinct oscillatory behavior between the two waveguides in the coupler region (right of the black line). (b) Polariton PL distribution $\Delta I=\left(I_{\mathrm{top}}-I_{\mathrm{bot}}\right) / I_{\mathrm{tot}}$. The red dashed line represents the results of theoretical fitting by Eq. (5).

coupler region in the linear regime and above threshold, respectively. The calculated dispersion along the coupler is shown in Fig. 1(f).

The experiments have been carried out with two photoluminescence (PL) setups, the first capable of both Fourierand real space emission detection while the second one was used for streak camera measurements. Excitation was provided using tuneable Ti:Sa-lasers with $10 \mathrm{ps}$ and $2 \mathrm{ps}$ pulse lengths set at the wavelength of a high energetic Bragg minimum of the microcavity for each structure. The excitation was mechanically chopped with a ratio of 1:12 to prevent sample heating. Additionally, a tomography technique using motorized lenses was implemented to allow for energy selective imaging. The pump spot was focused via a microscope objective with $\mathrm{NA}=$ 0.42 to a diameter of $\sim 3 \mu \mathrm{m}$.

As an example, we show the characterization of the incoupler with an excitation spot located at its center. The dispersion depicted in Fig. 1(d) was measured parallel to the incoupler at a low excitation density of $0.1 \mathrm{~mW}$ and fitted with an approach from Ref. ${ }^{40}$. This allows to extract a detuning of $-20 \mathrm{meV}$ with an exciton energy of $1.609 \mathrm{eV}$. An investigation of the non-linear regime in this structure, is represented in Fig. 1(e), showing polaritons above threshold being expelled from the small laser pump spot ${ }^{41}$ at a wave vector $\mathrm{k}_{\mathrm{x}} \approx 1.9 \mu \mathrm{m}^{-1}$. A detailed analysis of the emission intensity and energy as a function of the excitation power revealing a lasing threshold behavior and a continuous blueshift of $4 \mathrm{meV}$ is presented in the Supplemental Material, confirming polariton interaction ${ }^{42}$.

Let us now report on the main subject of the work, 

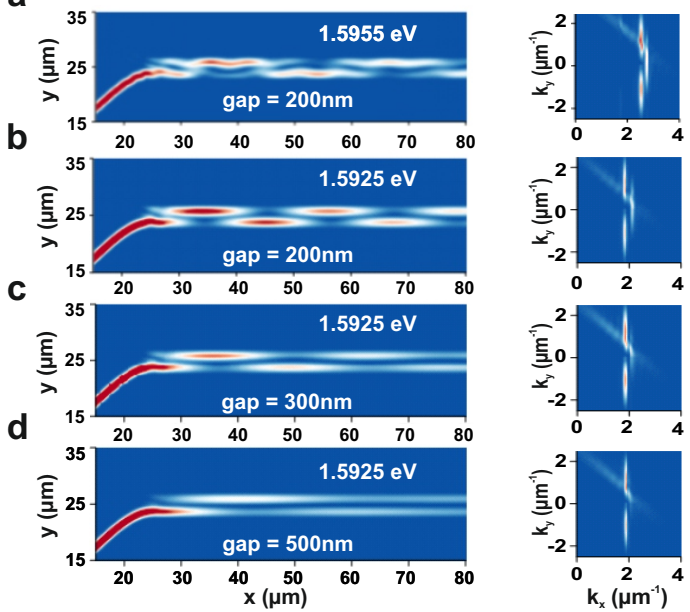

FIG. 3: Propagation dynamics of polaritons calculated within the model Eqs. 6 \& 7 for coupled waveguides with separation gap sizes of (a,b) $200 \mathrm{~nm}$, (c) $300 \mathrm{~nm}$, and (d) $500 \mathrm{~nm}$. While the dynamics for (a) is at an energy of $1.5955 \mathrm{eV}$ (b-d) are at $1.5925 \mathrm{eV}$. The insets on the right hand sides show the respective distribution in momentum space (on the parameter plane $k_{y}$ and $k_{x}$ ). Antisymmetric and symmetric modes of the coupler are visible.

namely the oscillations in co-directional couplers. To this end, Fig. 2(a) depicts a logarithmic color-coded real space image of the energy-resolved PL from two adjacent waveguides at an injection power of $1 \mathrm{~mW}$, corresponding to the propagating condensate regime for $E=1.5924-1.5930 \mathrm{eV}$. The gap between the two waveguides is $200 \mathrm{~nm}$ wide and the coupling area is $100 \mu \mathrm{m}$ long. The emission from the region of the excitation spot (on the bottom left incoupler) is attenuated by a neutral density filter, while the far propagating condensate is amplified to compensate for its disspative nature. A clear oscillation pattern is observed in the coupling region. In panel (b), the intensity distribution $\Delta I=\left(I_{\text {top }}-I_{\text {bot }}\right) / I_{\text {tot }}$ between the two waveguides, where $I_{\text {top }}, I_{\text {bot }}$ and $I_{\text {tot }}$ denote the intensities in the top, bottom and both waveguides, is presented. Therefore, $\Delta I=0$ corresponds to an equal distribution of the population. Due to continuous polariton decay, we observe an exponential spatial decay $\tau_{x}=80 \mu \mathrm{m}^{-1}$. From Fig. 2(b) a spatial oscillation period of $\sim 20 \mu \mathrm{m}$ is extracted.

The governing physics behind the observed oscillation dynamics can be understood within a slowly- varying amplitude approach for the modes of the coupled waveguides. Since the excitation occurs above condensation threshold, the ballistic polaritons have a well-defined frequency. Thus we focus on spatial dynamics along the propagation axis $x$ considering a monochromatic case. In the low-intensity limit the dynamics can be modelled via two coupled equations for amplitudes $A_{1,2}$ in the coupled waveguides

$$
\begin{aligned}
& i \frac{\partial}{\partial x} A_{1}(x)=-\kappa A_{1}(x)-J A_{2}(x), \\
& i \frac{\partial}{\partial x} A_{2}(x)=-\kappa A_{2}(x)-J A_{1}(x) .
\end{aligned}
$$

where $J \equiv J^{\prime}+i J^{\prime \prime}$ is the complex coupling constant which is governed by the width and depth of the gap between the coupled waveguides. The complex wavevector $\kappa=k+i \alpha$ characterizes the propagation and damping of guided modes in separated waveguides (in the following $\left.\alpha>J^{\prime \prime}>0\right)$.

In order to find the super-modes of the coupler we look for a solution of Eqs. (1) and (2) in the form $A_{1,2}(x)=a_{1,2} \exp (i \beta x)$. By diagonalizing the system one finds two eigenmodes, namely symmetric $a_{1}=a_{2}$ and antisymmetric $a_{1}=-a_{2}$ one, with the propagation constants $\beta_{+}=\kappa+J^{\prime}+i J^{\prime \prime}$ and $\beta_{-}=\kappa-J^{\prime}-i J^{\prime \prime}$, respectively. It is worth mentioning that, due to the imaginary parts of the coupling constants, the damping rates $\operatorname{Im}\left(\beta_{ \pm}\right)=\alpha \pm J^{\prime \prime}$ of these two modes are different. More precisely, the antisymmetric mode has smaller propagation losses. If only one waveguide is excited (with an amplitude $A$ ), the analytical solution for the mode dynamics can be easily found as

$$
\begin{aligned}
& A_{1}(x)=A e^{i k x-\alpha x} \\
& \quad \times\left(\cos \left(J^{\prime} x\right) \cosh \left(J^{\prime \prime} x\right)-i \sin \left(J^{\prime} x\right) \sinh \left(J^{\prime \prime} x\right)\right), \\
& A_{2}(x)=A e^{i k x-\alpha x} \\
& \quad \times\left(i \sin \left(J^{\prime} x\right) \cosh \left(J^{\prime \prime} x\right)-\cos \left(J^{\prime} x\right) \sinh \left(J^{\prime \prime} x\right)\right) .
\end{aligned}
$$

From the analytical solution $(3,4)$ it is easy to derive an expression for the intensity distribution in the coupler

$$
\Delta I=\left(I_{1}-I_{2}\right) / I_{\mathrm{tot}}=\cos \left(2 J^{\prime} x\right) / \cosh \left(2 J^{\prime \prime} x\right) .
$$

This solution has a form of damped oscillations where polaritons transfer between the two channels with a spatial period $\pi / J^{\prime}=21.36 \mu \mathrm{m}$ and a damping coefficient given by the imaginary part of the coupling constant $2 J^{\prime \prime}=0.021 \mu m^{-1}$ [see Fig. $2(\mathrm{~b})$ ]. It is worth mentioning, that, due to the etching of the Bragg mirror between two channels, the effect of local losses becomes comparable with the polariton tunnelling dynamics and thus the imaginary part of the coupling cannot be neglected. For a non-zero imaginary part $J^{\prime \prime}>0$, the symmetric mode decays faster and at propagation distances of the order of $1 / J^{\prime \prime}$ it becomes much less intensive than the antisymmetric mode. This suppresses the mode beating at large propagation distances.

To underpin this rather qualitative analysis with a more thorough theoretical study, we performed numerical calculations in the frame of the mean-field model for $2 \mathrm{D}$ intracavity photons coupled strongly to the QW excitons $^{6,7}$. 
Neglecting polarization effects one obtains two coupled Schrödinger equations for the photonic field $\Psi_{\mathrm{C}}$ and coherent excitons $\Psi_{\mathrm{E}}$ given as

$$
\begin{aligned}
\partial_{t} \Psi_{\mathrm{C}}- & \frac{i \hbar}{2 m_{\mathrm{c}}} \nabla_{x, y}^{2} \Psi_{\mathrm{C}}+i V(x, y) \Psi_{\mathrm{C}} \\
& +\left[\gamma_{\mathrm{c}}-i\left(\omega_{\mathrm{p}}-\delta\right)\right] \Psi_{\mathrm{C}}=i \Omega_{\mathrm{R}} \Psi_{\mathrm{E}}+\Psi_{\mathrm{C}}(x, y) e^{i k_{\mathrm{p}} x},
\end{aligned}
$$

$$
\partial_{t} \Psi_{\mathrm{E}}-\frac{i \hbar}{2 m_{\mathrm{E}}} \nabla_{x, y}^{2} \Psi_{\mathrm{E}}+\left[\gamma_{\mathrm{e}}-i \omega_{\mathrm{p}}\right] \Psi_{\mathrm{E}}=i \Omega_{\mathrm{R}} \Psi_{\mathrm{C}}
$$

The complex amplitudes are obtained through a standard averaging procedure of the related creation or annihilation operators. $\gamma_{c}$ and $\gamma_{e}$ denote the cavity photon damping and dephasing rate of excitons, respectively. We note that, for cryogenic sample temperatures, the excitonic dephasing time can be comparable with the life-time of intracavity photons. Thus, without loss of generality, they are assumed to be equal $\hbar \gamma_{c}=\hbar \gamma_{e}=0.01 \mathrm{meV}$. The effective photon mass in the planar region is given by $m_{\mathrm{c}}=36.13 \times 10^{-6} m_{\mathrm{e}}$ where $m_{\mathrm{e}}$ is the free electron mass. The effective mass of excitons is $m_{\mathrm{E}} \approx 10^{5} m_{\mathrm{C}}$. $\Omega_{\mathrm{R}}$ is the Rabi frequency which defines the Rabi splitting $2 \hbar \Omega_{\mathrm{R}}=13.9 \mathrm{meV}$. The photon - exciton detuning is given by the parameter $\hbar \delta=\hbar \omega_{c}-\hbar \omega_{e}=-20 \mathrm{meV}$ where $\omega_{\mathrm{c}}$ is the cavity resonance frequency and $\omega_{e}$ is the excitonic resonance. Theoretical details regarding the chosen potential $V(x, y)$ and the excitation modeling can be found in the Supplemental Material.

Figs. 3(a) and (b) show examples of oscillation dynamics for two slightly different frequencies of the wavepackets. These oscillations are governed by interference of the symmetric and the antisymmetric modes of the coupler, which is clearly visible in the two-dimensional spectrum, shown in the insets to Figs 3 . The insets in each panel represent the momentum space distribution of the propagating polaritons. The substantial difference in the period of spatial oscillations can be explained by a non-equidistant momentum splitting between the above mentioned symmetric and antisymmetric modes within the dispersion [see Fig. 1(f)]. Further examples in Figs. 3(c)-(d) show propagation dynamics in which the coupling strength is continuously weakened due to decreased wavefunction overlap via increased gap width. A clear change in the oscillation pattern is observed. Note that, due to pronounced dissipative effects within the gap between waveguides, the antisymmetric mode, which has the lowest overlap with the gap region, dominates the spectrum for larger gaps and, as a result, oscillations disappear [see Fig. 3(d)].

Now, in order to demonstrate the polariton dynamics, we have performed energy- and time-resolved streak camera measurements using two devices with gap sizes of $200 \mathrm{~nm}$ and $500 \mathrm{~nm}$. We use a streak camera with a time resolution of $10 \mathrm{ps}$. Due to the fast dynamics of polaritons in this sample, the polariton propagation is shown up to $30 \mathrm{ps}$ here. The respective intensity patterns are
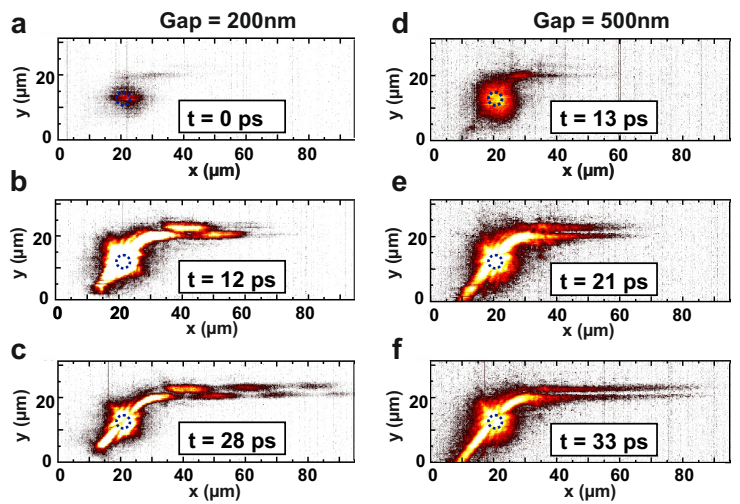

FIG. 4: a-c) Time-resolved propagation of the polariton propagation in coupler devices with a gap size of (a-c) $200 \mathrm{~nm}$ and (d-f) $500 \mathrm{~nm}$ for three different times during the propagation. While (a-c) show a clean oscillation between the two waveguides, no oscillation is visible in (d-f). The pump area is indicated by a blue dashed circle.

plotted in Figs. 4(a)-(c) and (d)-(f).

In Fig. $4(\mathrm{a})$ at $\mathrm{t}=0 \mathrm{ps}$, we observe the laser excitation spot on the lower left input coupler from where polaritons are repulsively expelled into the coupling region. At $\mathrm{t}=12 \mathrm{ps}$, the polaritons have finished the first full oscillation. After approximately $30 \mathrm{ps}$, the polariton population has dissipated after a propagation length of $100 \mu \mathrm{m}$ underlining the excellent quality of the patterned microcavity structure.

Figs. 4(d)-(f) shows the temporal evolution in a system with a much larger gap of $500 \mathrm{~nm}$. In this case, while there is some evanescent coupling to the upper waveguide, no pronounced oscillatory behavior is observed, again in excellent agreement with the theory presented in Fig. 3.

From the experiments as well as from the theoretical model, we can infer that a variation in the coupling strength ultimately allows for a change of the oscillation period. This can be used to choose the outputport of the exciton-polariton flow due to a specifically tailored channel length and etch depth, similar to coupling behavior previously demonstrated for surface plasmon polaritons ${ }^{43,44}$. Fig. 5 shows the results of the experiments. We have used similar couplers with $200 \mathrm{~nm}$ and $300 \mathrm{~nm}$ gap size respectively, but with a reduced coupler region length of $20 \mu \mathrm{m}$. While (a) shows the polariton flow leaving predominantly through the left outcoupler, the larger gap (weaker coupling and larger oscillation length) in (b) shows predominant coupling to the right arm. In (c) the normalized line-profiles are plotted for Figs. $5(\mathrm{a}, \mathrm{b})$ at $x=28 \mu \mathrm{m}$. Another way of changing the output port is by changing the energy of the polariton condensate. The emissions of a condensate for three different energies ranging from $E=1.592-1.594 \mathrm{eV}$ are shown in Figs. 5(d-f). Changing the condensate propagation energy by $2 \mathrm{meV}$, a change of the intensity from left to right can be observed. This shift is underlined by 

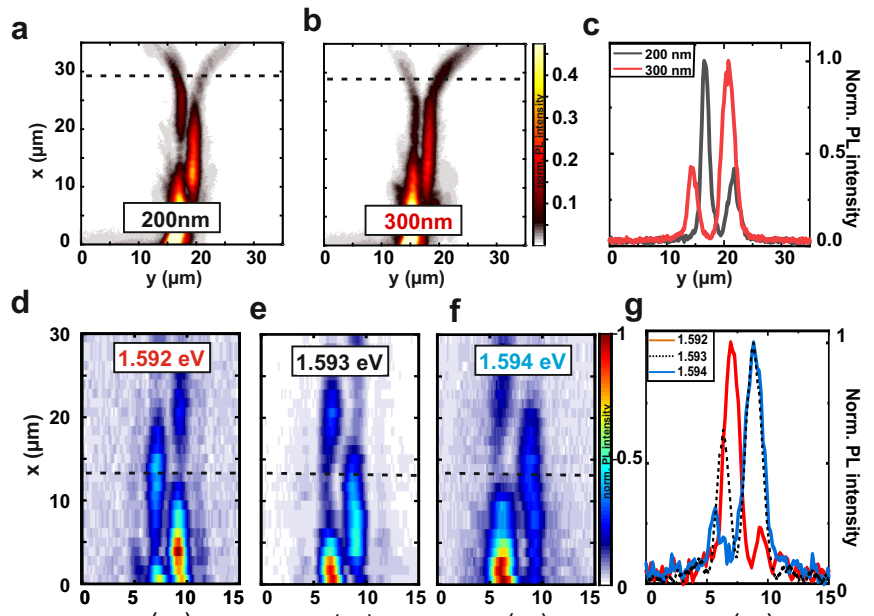
g

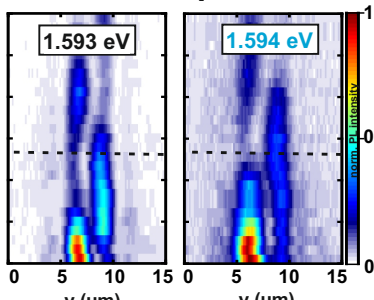

h

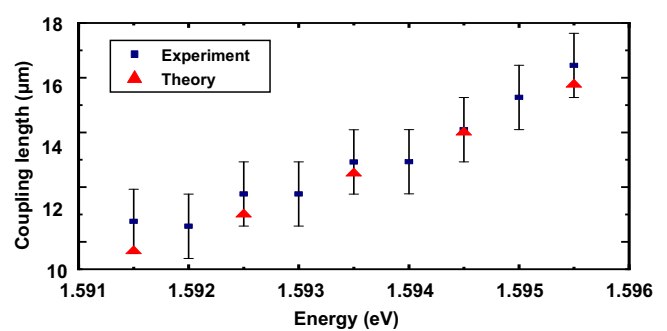

FIG. 5: Routing of a polariton condensate via a different coupling length (a-c) and different propagation energy (d-g). (Note that the plot orientation has been turned by $90^{\circ}$ for visual clarity). A coupler with a length of the coupling region of of $20 \mu \mathrm{m}$ and a gap size of (a) $200 \mathrm{~nm}$ and (b) $300 \mathrm{~nm}$ is shown. (c) Line profiles of the normalized intensities at $x=$ $28 \mu \mathrm{m}$ for (a) and (b). PL Emission for different energies (d$\mathrm{f}$ ) in a fixed region of the coupler is shown. (g) Normalized PL intensity line profiles of the emission at $x=13 \mu \mathrm{m}$. (h) Experimentally extracted coupling lengths as a function of the propagation energy and numerically calculated values.

the line profiles in Fig. 5(g). The detailed change of experimental coupling lengths in excellent agreement with the numerically calculated values for Figs. 5(d-f) are depicted in the Fig. 5(h).

Therefore, we have shown that this device configuration allows for co-directional routing to a predetermined exit-port via a Josephson-like oscillation effect in real space.

In conclusion, we have demonstrated the possibility for passive polariton routing, which is easily scalable and integratable to large polariton based logic networks. We evidenced this by a precise control of the lithographically engineered photonic landscape, which allows for the observation of these oscillations in real space between polaritonic waveguides. Such detailed tailoring of the flow of quantum fluids of light paves the way to harness their non-linearity in next generation photonics. Furthermore, the basic understanding of the coupling of polariton waveguides ${ }^{45,46}$ is the necessary foundation for larger coupled waveguide arrays, comparable to those that have been implemented for the demonstration of topological waveguiding effects ${ }^{47,48}$. In this respect, our work opens a new route, to use polariton waveguides for polariton logic as well as for topological devices involving nonlinearity, gain, interactions and coherence, inherent to the polariton system.

\section{Acknowledgments}

The Würzburg and Jena group acknowledge financial support within the DFG projects SCHN1376/3-1, PE 523/18-1 and KL3124/2-1 . The Würzburg group is grateful for support from the state of Bavaria and within the Würzburg-Dresden Cluster of Excellence ct.qmat. S.H. also acknowledges support by the EPSRC Hybrid Polaritonics grant (EP/ M025330/1). The Würzburg group wants to thank Hugo Flayac for inspiring discussions in the early stage of this work.

The Madrid group acknowledges financial support of the Spanish MINECO Grant MAT2017-83722-R. E.R. acknowledges financial support from a Spanish FPI Scholarship No. BES-2015-074708. I.A.Shelykh acknowledges financial support of Icelandic Science Foundation (project Hybrid polaritonics). I.A.Shelykh and A.Yulin acknowledge Ministry of Science and Higher Education of the Russian Federation (Megagrant number 14.Y26.31.0015).
1 D.A.B. Miller, Nat. Photonics 4, 3 (2010).

${ }^{2}$ Z. Lu, H. Yun, Y. Wang, Z. Chen, F. Zhang, N. A. F. Jaeger, and L. Chrostowski, Opt. Express 23, 3795 (2015).

3 C. Weisbuch, M. Nishioka, A. Ishikawa, and Y. Arakawa, Phys. Rev. Lett. 69, 3314 (1992).

4 J. Kasprzak, M. Richard, S. Kundermann, A. Baas, P. Jeambrun, J. M. J. Keeling, F. M. Marchetti, M. H. Szymanska, R. Andre, J. L. Staehli, V. Savona, P. B. Littlewood, B. Deveaud, and L. S. Dang, Nature 443, 409 (2006).

5 A. Imamoglu, R. J. Ram, S. Pau, and Y. Yamamoto, Phys. Rev. A 53, 4250 (1996).
${ }^{6}$ I. Carusotto and C. Ciuti, Rev. Mod. Phys. 85, 299 (2013).

7 A. Amo, J. Lefrere, S. Pigeon, C. Adrados, C. Ciuti, I. Carusotto, R. Houdre, E. Giacobino, and A. Bramati, Nat. Phys. 5, 805 (2009).

8 B. Nelsen, G. Liu, M. Steger, D.W. Snoke, R. Balili, K. West, and L. Pfeiffer, Phys. Rev. X 3, 041015 (2013).

9 E. Wertz, A. Amo, D.D. Solnyshkov, L. Ferrier, T.C.H. Liew, D. Sanvitto, P. Senellart, I. Sagnes, A. Lemaître, A. V. Kavokin, G. Malpuech, and J. Bloch, Phys. Rev. Lett. 109, 216404 (2012).

10 M. Vladimirova, S. Cronenberger, D. Scalbert, K. V. Kavokin, A. Miard, A. Lemaître, J. Bloch, D. Solnyshkov, G. 
Malpuech, and A. V. Kavokin, Phys. Rev. B 82, 075301 (2010).

11 G. Muñoz-Matutano, A. Wood, M. Johnsson, X. Vidal, B. Q. Baragiola, A. Reinhard, A. Lemaître, J. Bloch, A. Amo, G. Nogues, B. Besga, M. Richard and T. Volz, Nat. Mater. 18, 213218 (2019)

12 A. Delteil, T. Fink, A. Schade, S. Höfling, C. Schneider and A. Imamoglu, Nat. Mater. 18, 219222 (2019).

13 D. Sanvitto, and S. Kena-Cohen, Nat. Mater. 15, 1061 (2016).

14 T. C. H. Liew, A. V. Kavokin, and I. A. Shelykh, Phys. Rev. Lett. 101, 016402 (2008)

15 A. Amo, T. C. H. Liew, C. Adrados, R. Houdré, E. Giacobino, A. V. Kavokin, and A. Bramati, Nat. Photonics 4, 361 (2010).

16 C. Anton, T. C. H. Liew, G. Tosi, M. D. Martń, T. Gao, Z. Hatzopoulos, P. S. Eldridge, P. G. Savvidis, and L. Viña, Appl. Phys. Lett., 101, 261116 (2012)

17 T. Gao, P. S. Eldridge, T. C. H. Liew, S. I. Tsintzos, G. Stavrinidis, G. Deligeorgis, Z. Hatzopoulos, and P. G. Savvidis, Phys. Rev. B 85, 235102 (2012).

18 C. Anton, T. C. H. Liew, J. Cuadra, M. D. Martin, P. S. Eldridge, Z. Hatzopoulos, G. Stavrinidis, P. G. Savvidis, and L. Viña, Phys. Rev. B 88, 245307 (2013)

19 D. Ballarini, M. De Giorgi, E. Cancellieri, R. Houdr; E. Giacobino, R. Cingolani, A. Bramati, G. Gigli, and D. Sanvitto, Nat. Commun. 4, 1778 (2013).

${ }^{20}$ H. S. Nguyen, D. Vishnevsky, C. Sturm, D. Tanese, D. Solnyshkov, E. Galopin, A. Lemaître, I. Sagnes, A. Amo, G. Malpuech, and J. Bloch, Phys. Rev. Lett. 110, 236601 (2013).

21 H. Suchomel, S. Brodbeck, T. C. H. Liew, M. Amthor, M. Klaas, S. Klembt, M. Kamp, S. Höfling and C. Schneider, Sci. Rep. 7, 5114 (2017).

${ }^{22}$ K. Winkler, H. Flayac, S. Klembt, A. Schade, D. Nevinskiy, M. Kamp, C. Schneider, and S. Höfling, Phys. Rev. B 95, 201302(R) (2017).

${ }^{23}$ R. Su, J. Wang, J. Zhao, J. Xing, W. Zhao, C. Diederichs, T. C. H. Liew and Qihua Xiong, Sci. Adv. 4, eaau0244 (2018).

24 A. V. Zasedatelev, A. V. Baranikov, D. Urbonas, F. Scafirimuto, U. Scherf, T. Stöferle, R. F. Mahrt, P. G Lagoudakis, Nat. Photonics 13, 378383 (2019).

${ }^{25}$ H. Flayac, and I. G. Savenko, Appl. Phys. Lett. 103, 201105 (2013).

${ }^{26}$ F. Marsault, H.S. Nguyen, D. Tanese, A. Lemaître, E. Galopin, I. Sagnes, A. Amo, and J. Bloch, Appl. Phys. Lett. 107, 201115 (2015)

27 B. Josephson, Phys. Lett. 7, 251 (1962).

28 P. W. Anderson, Rev. Mod. Phys. 38, 298 (1966).

29 F. S. Cataliotti, S. Burger, C. Fort, P. Maddaloni, F. Mi- nardi, A. Trombettoni, A. Smerzi, and M. Inguscio, Science 293, 843 (2001)

${ }^{30}$ M. Albiez, R. Gati, J. Foelling, S. Hunsmann, M. Cristiani, und M. K. Oberthaler, Phys. Rev. Lett. 95, 010402 (2005).

${ }^{31}$ R. Gati, M. Albiez, J. Foelling, B. Hemmerling. und M. Oberthaler, Appl. Phys. B 82, 207 (2006).

32 R. Gati, und M. K. Oberthaler, J. Phys. B 40, R61 (2007).

${ }^{33}$ S. Levy, E. Lahoud, I. Shomroni, und J. Steinhauer, Nature 449, 579 (2007).

34 A. Smerzi, S. Fantoni, S. Giovanazzi, and S. R. Shenoy, Phys. Rev. Lett 79, 4950 (1997).

35 A. J. Leggett, Rev. Mod. Phys. 73, 307 (2001).

${ }^{36}$ K. G. Lagoudakis, B. Pietka, M. Wouters, R. André, and B. Deveaud-Plédran, Phys. Rev. Lett. 105, 120403 (2010).

37 M. Abbarchi, A. Amo, V. G. Sala, D. D. Solnyshkov, H. Flayac, L. Ferrier, I. Sagnes, E. Galopin, A. Lemaître, G. Malpuech, and J. Bloch, Nat. Phys. 9, 275 (2013).

38 A. F. Adiyatullin, M. D. Anderson, H. Flayac, M. T. Portella-Oberli, F. Jabeen, C. Ouellet-Plamondon, G. C. Sallen and B. Deveaud, Nature Commun. 8, 1329 (2017).

39 C. Schneider, K. Winkler, M. D. Fraser, M. Kamp, Y. Yamamoto, E. A. Ostrovskaya and S. Höfling, Rep. Prog. Phys. 80, 1 (2016).

40 A. I. Tartakovskii and V. D. Kulakovskii and A. Forchel and J. P. Reithmaier, Phys. Rev. B 57, R6807 (1998).

41 E. Wertz, L. Ferrier, D. D. Solnyshkov, R. Johne, D. Sanvitto, A. Lemaître, I. Sagnes, R. Grousson, A. V. Kavokin, P. Senellart, G. Malpuech, and J. Bloch, Nat. Phys. 6, 860864 (2010).

42 C. Ciuti, V. Savona, C. Piermarocchi, A. Quattropani, and P. Schwendimann, Phys. Rev. B 58, 7926 (1998).

43 M.-Y. Pan, E.-H. Lin, L. Wang, and P.-K. Wei, Nanoscale Res. Lett. 9, 430 (2014).

${ }^{44}$ Y. Zhang, Y. Xu, C. Tian, Q. Xu, X. Zhang, Y. Li, X. Zhang, J. Han, and W. Zhang, Photon. Res. 6, 18 (2018).

${ }^{45}$ M. Klaas, J. Beierlein, E. Rozas, S. Klembt, H. Suchomel, T. H. Harder, K. Winkler, M. Emmerling, H. Flayac, M.D. Martín, L. Viña, S. Höfling, and C. Schneider Appl. Phys. Lett. 114, 061102 (2019)

46 E. Rozas, J. Beierlein, A.Yulin, M. Klaas, H. Suchomel, O. Egorov, I.A. Shelykh, U. Peschel, C. Schneider, S. Klembt, S. Höfling, M.D. Martín, and L. Viña, Adv. Optical Mater. (2020) DOI: 10.1002/adom.202000650.

47 A. Blanco-Redondo, I. Andonegui, M. J. Collins, Gal Harari, Y. Lumer, M. C. Rechtsman, B. J. Eggleton, and M. Segev Phys. Rev. Lett. 116, 163901 (2016).

48 S. Weimann, M. Kremer, Y. Plotnik, Y. Lumer, S. Nolte, K. G. Makris, M. Segev, M. C. Rechtsman, and A. Szameit Nat. Mater. 16, 433438 (2017). 